\title{
Comparison of histologic subtype and growth pattern in intraductal papillary-mucinous carcinoma of the pancreas
}

\author{
YUICHI SANADA ${ }^{1,2}$, SATOKO KUNITA $^{3}$ and KAZUHIRO YOSHIDA ${ }^{2}$ \\ ${ }^{1}$ Department of Surgery, Saiseikai Kure Hospital, Hiroshima; ${ }^{2}$ Department of Surgical Oncology, \\ Research Institution for Radiation Biology and Medicine, Hiroshima University, Hiroshima; \\ ${ }^{3}$ Department of Internal Medicine, Saiseikai Kure Hospital, Hiroshima, Japan
}

Received September 25, 2007; Accepted November 19, 2007

\begin{abstract}
The purpose of this study was to compare histologic subtype and growth pattern, including invasion and intraductal spread to branch ducts, in main duct-type intraductal papillary-mucinous carcinoma (IPMC) by histopathology and immunohistochemistry. Five surgically resected samples of main duct-type IPMC from five patients, were studied. Main lesions, invasive components, and adjacent secondary ducts were examined microscopically. We performed immunohistochemistry with monoclonal mucin 2 (MUC2) and polyclonal orotate phosphoribosyltransferase (OPRT) antibodies. Three cases showed adenoma components in the main duct. Two of these showed intestinal-type accompanied by intraductal spread to branch ducts, neoplastic changes in branch ducts consisting of high-grade pancreatic intraepithelial neoplasia-like ducts positive for MUC2, and ducts filled with arborizing neoplastic cells, resembling pancreatobiliary type. The other case showed gastric-type adenoma and intestinal-type carcinoma in situ (CIS) in the main duct, with minimal tubular invasion. The two remaining cases showed no adenoma components in the main duct, but showed abrupt transition from normal epithelium to CIS (pancreatobiliary type or oncocytic type) and massive invasion diffusely positive for OPRT. These results suggest that IPMC with adenoma components in the main duct undergoes intraductal spread to branch ducts and has low malignant potential. The progression of one subtype to another is associated with intraductal spread of intraductal papillary-mucinous neoplasm (IPMN). However, IPMC without adenoma components is associated with marked invasion.
\end{abstract}

Correspondence to: Dr Yuichi Sanada, Department of Surgical Oncology, Research Institution for Radiation Biology and Medicine, Hiroshima University, 1-2-3 Kasumi, Minami-ku, Hiroshima 7348551, Japan

E-mail: ysanadasurg@hotmail.com

Key words: intraductal papillary-mucinous carcinoma, orotate phosphoribosyltransferase, mucin 2 , subtype

\section{Introduction}

At a meeting of international experts on pancreatic precursor lesions held in 2003, a consensus classification of the subtypes of intraductal papillary-mucinous neoplasm (IPMN) of the pancreas was established on the basis of morphologic features and mucin (MUC) expression, including that of MUC1, MUC2, and MUC5AC (1). The four subtypes are gastric, intestinal, pancreatobiliary and oncocytic. This subclassification allows for more detailed analyses of the clinicopathologic features of IPMN. Recent histopathologic research has shown that the malignant potential of IPMN depends on the subtype. For example, gastric-type IPMN usually presents as relatively small lesions with low-grade atypia corresponding predominantly to adenoma, whereas intestinal-type IPMN often presents as large lesions involving the main duct, with moderate to marked atypia corresponding to the borderline category or to carcinoma in situ (CIS) $(2,3)$. Most pancreatobiliary- and oncocytic-type IPMNs show marked atypia corresponding to that of CIS (4). Some studies have shown that IPMN is often composed of a combination of more than one type, including gastric and intestinal types $(1,5)$, and gastric and pancreatobiliary types $(6)$, with a transition from one to the other. We recently identified a case of main duct-type IPMN consisting of gastric-type adenoma with an intestinal-type borderline lesion (unpublished data). The combined features of more than one subtype suggest that the stepwise progression of IPMN is associated with a change of subtype and that one subtype can be a precursor of another. However, in intraductal papillary-mucinous carcinoma (IPMC), including invasive carcinoma, the relation between histologic subtype and growth pattern in the intraductal or invasive components has rarely been reported.

In the present study, we analyzed five cases of main ducttype IPMC and performed a histopathologic comparison of the subtype distribution in the main lesion and growth pattern, including invasive components and cancerization of branch ducts by MUC2 immunohistochemistry (IHC). We previously reported that orotate phosphoribosyltransferase (OPRT) expression is increased in high-grade pancreatic intraepithelial neoplasia (PanIN) $(7,8)$. To better understand the features of neoplastic changes in IPMC, we also performed IHC for OPRT. 
Table I. Clinicopathologic features of the patients with IPMC.

\begin{tabular}{lccccccc}
\hline $\begin{array}{l}\text { Case } \\
\text { no. }\end{array}$ & $\begin{array}{c}\text { Age } \\
\text { (years) }\end{array}$ & $\begin{array}{c}\text { Sex } \\
(\mathrm{M} / \mathrm{F})\end{array}$ & $\begin{array}{c}\text { Clinical } \\
\text { presentation }\end{array}$ & Enzyme & $\begin{array}{c}\text { Location } \\
\text { in pancreas }\end{array}$ & $\begin{array}{c}\text { Size } \\
\text { (diameter) }\end{array}$ & $\begin{array}{c}\text { Surgical } \\
\text { procedure }\end{array}$ \\
\hline 1 & 70 & F & Back pain & $(+)$ & Head & $4.5 \mathrm{~cm}$ & PD \\
2 & 63 & F & Back pain & $(-)$ & Body & $3.0 \mathrm{~cm}$ & DP \\
3 & 69 & F & None & $(-)$ & Head & $2.2 \mathrm{~cm}$ & PD \\
4 & 87 & M & None & $(+)$ & Body & $3.3 \mathrm{~cm}$ & DP \\
5 & 64 & M & Back Pain & $(-)$ & Head & $2.8 \mathrm{~cm}$ & PD \\
\hline
\end{tabular}

Enzyme, presence (+), absence (-) of serum levels of pancreatic enzymes; PD, pancreatoduodenectomy; DP, distal pancreatectomy.

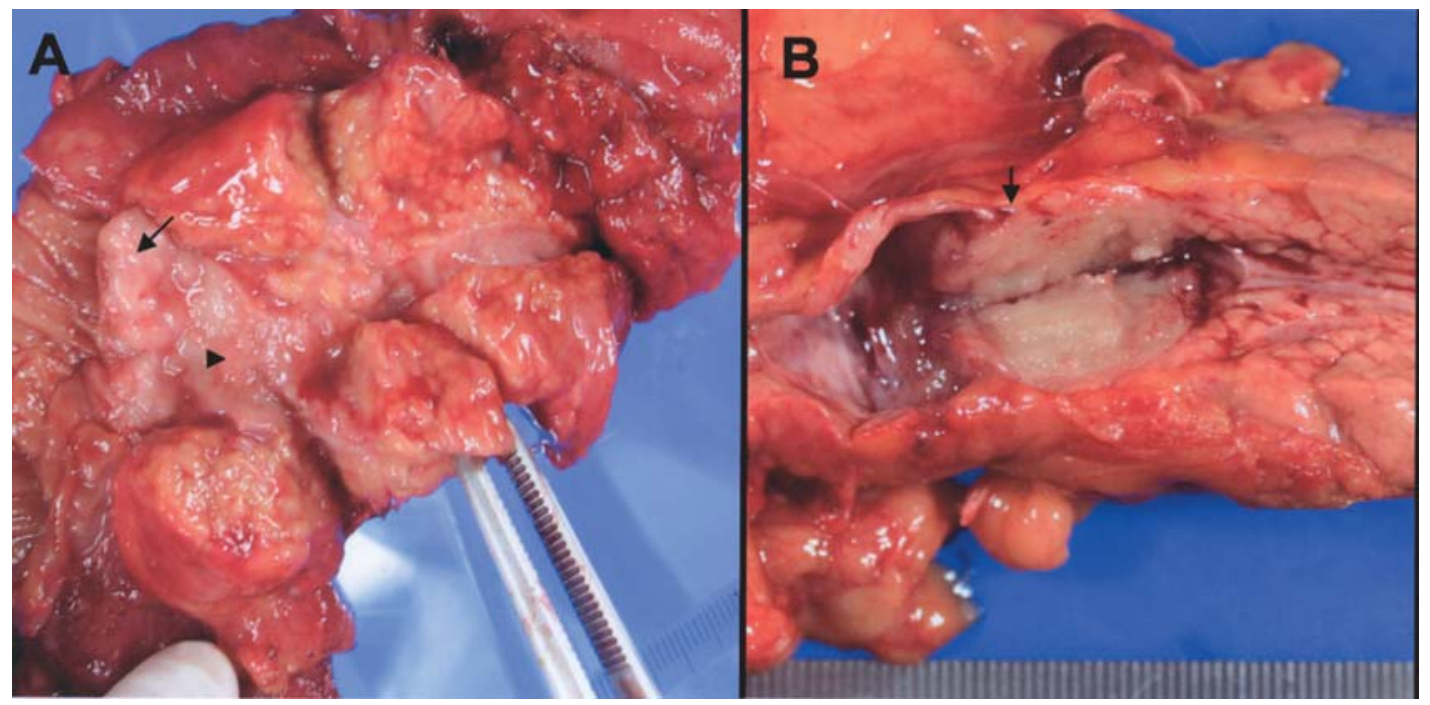

Figure 1. Gross appearance of representative cut sections. (A) Case 1. Main pancreatic duct filled with soft papillary tumors. Flat components are observed in the peripheral area of the tumor (arrowhead). (B) Case 4. A firm white mass in the markedly dilated main pancreatic duct is accompanied by thickening of the pancreatic parenchyma around the tumor (arrow).

\section{Materials and methods}

Cases. The minimum criterion for inclusion of a tumor in this study was either i) cystic dilation of the main pancreatic duct detected radiographically or on a macroscopic examination of resected specimens, ii) marked (macroscopically visible) intraductal mass formation with mucin production in the main pancreatic duct, or iii) the presence of carcinoma (noninvasive or invasive) components detected histologically. Five resected specimens that fulfilled these criteria were identified on file in the Department of Surgical Oncology at Hiroshima University and the Department of Surgery at Saiseikai Kure Hospital during the period 1999 through 2007. The patients were two men and three women, aged 6487 years (mean, 70.6). Three patients presented with back pain. Two patients showed elevated serum levels of pancreatic enzymes. The surgical procedures consisted of pancreatoduodenectomy $(\mathrm{n}=3)$ and distal pancreatectomy $(n=2)$ (Table I).

Histologic evaluation. All specimens were cut transversally with the main pancreatic duct. The size of the tumor in the main pancreatic duct was measured. In addition, the parenchyma surrounding the main tumor was examined macroscopically. Specimens were formalin-fixed, paraffinembedded, and cut into $4-\mu \mathrm{m}$ sections. Sections were stained with hematoxylin and eosin and examined by microscopy. The grade of the tumor in the main pancreatic duct was determined on the basis of the current World Health Organization definition and grading of IPMNs. In IPMN adenoma, the papillae were architecturally simple, and the epithelium was composed of tall columnar cells with uniform basally oriented nuclei with no or slight atypia. IPMN with moderate dysplasia was classified as borderline; the epithelium showed no more than moderate loss of polarity, nuclear crowding, nuclear enlargement, pseudostratification, and nuclear hyperchromatism. IPMN with severe dysplastic epithelial change was considered to be carcinoma; cribriform growth, atypical mitoses, and the budding off of small clusters of epithelial cells into the lumen indicated carcinoma. Histologic tumor subtype was classified according to the consensus criteria of Furukawa et al (1). Gastric-type IPMN consisted of cells resembling gastric foveolae. Intestinal-type IPMN resembled intestinal villous 
Table II. Histopathologic features of IPMCs.

\begin{tabular}{|c|c|c|c|c|c|c|c|c|}
\hline \multirow{2}{*}{$\begin{array}{l}\text { Case } \\
\text { no. }\end{array}$} & \multicolumn{3}{|c|}{ Main duct tumor } & \multicolumn{3}{|c|}{ Branch ducts (histologic features) } & \multirow[t]{2}{*}{ Invasion } & \multirow{2}{*}{$\begin{array}{c}\text { Invasion } \\
\text { type }\end{array}$} \\
\hline & Adenoma & Grade & Subtype & Cancerization & Low-PanIN & High-PanIN & & \\
\hline 1 & $(+)$ & $\mathrm{Ca}$ & $\mathrm{G}+\mathrm{IN}$ & $(+)$ & $(+)$ & $(-)$ & $(+) \min$ & Tub \\
\hline 2 & $(-)$ & $\mathrm{Ca}$ & Oncocytic & $(+)$ & $(+)$ & $(-)$ & $(+)$ & Tub+Muc \\
\hline 3 & $(+)$ & Border & IN & $(-)$ & $(-)$ & $(+)$ & $(-)$ & $*$ \\
\hline 4 & $(-)$ & $\mathrm{Ca}$ & PB & $(+)$ & $(+)$ & $(-)$ & $(+)$ & Tub \\
\hline 5 & $(+)$ & Border & IN & $(+)$ & $(+)$ & $(+)$ & $(+) \min$ & Tub \\
\hline
\end{tabular}

Adenoma, presence (+), absence (-) of adenoma components; Grade, highest histologic grade of the tumor (Ca, carcinoma; Border, borderline lesion); Subtype, histologic subtype of the tumor (G, gastric type; IN, intestinal type; PB, pancreatobiliary type; Oncocytic: oncocytic type); Cancerization, presence (+), absence (-) of cancerization; Low-PanIN, presence (+), absence (-) of low-grade PanIN; High-PanIN, presence $(+)$, absence (-) of high-grade PanIN; Invasion, presence (+), absence (-) of invasive components (min, minimal); tub, tubular; muc, mucinous.

neoplasms with tall columnar epithelial cells and spindleshaped nuclei. Pancreatobiliary-type IPMN consisted of cells resembling cholangiopapillary neoplasia and showed complex, thin, branching papillae. Oncocytic-type IPMN consisted of cells with abundant, intensely eosinophilic cytoplasm and showed complex thick papillae with intraepithelial lumina. In IPMNs composed of more than one subtype, the predominant subtype was identified, and the histologic grade corresponding to each subtype was determined. Neoplastic change of the branch ducts around the main tumor was examined in each specimen. PanIN was classified as follows: PanIN-1A showed flat epithelial lesions composed of tall columnar cells with basally located nuclei; PanIN-1B showed epithelial lesions with a papillary, micropapillary, or basally pseudostratified architecture; PanIN-2 showed epithelial lesions that were mostly papillary and showed nuclear abnormalities including some loss of polarity, nuclear crowding, pseudostratification, or hyperchromatism; and PanIN-3 showed cribriforming, as evidenced by budding off of small clusters of epithelial cells into the lumen, and luminal necrosis. PanIN-1A and -1B were classified as low-grade, and PanIN-2 and -3 were classified as high-grade. Cancerization of the branch ducts was defined as an abrupt transition from normal epithelial cells to a neoplastic papillary epithelium showing intraluminal nodular growth or intraductal neoplastic cells lining the entire epithelium of the branch duct. Invasive components adjacent to the intraductal carcinoma were also examined. Minimal invasion was defined as the presence of lesions with only a few tumor glands communicating with the intraductal carcinoma components. Invasion was classified as tubulartype or mucinous-type. IHC for MUC2 and OPRT was carried out with a monoclonal anti-MUC2 antibody (1:150, Novocastra Laboratories, Newcastle upon Tyne, UK) and a polyclonal anti-OPRT antibody (1:1000, Taiho, Tokyo, Japan), and Dako LSAB Kit (Dako, Carpinteria, CA, USA). Paraffin-embedded sections were deparaffinized in xylene and rehydrated through a graded ethanol series. After the blockage of endogenous peroxidase activity with $3 \% \mathrm{H}_{2} \mathrm{O}_{2}$ in methanol for $10 \mathrm{~min}$, sections were incubated with the primary antibody for $8 \mathrm{~h}$ at $4^{\circ} \mathrm{C}$, followed by sequential 10 min incubations with biotinylated anti-rabbit $\mathrm{IgG}$ and peroxidase-labeled streptavidin. Staining was completed with a 10-min incubation in substrate-chromogen solution. Sections were counterstained in $0.1 \%$ hematoxylin. The results of the antibody staining were graded according to the percentage of stained target cells. Staining was considered focally positive (+) if at least $10 \%$ of the cells were stained and diffusely positive $(++)$ if at least $50 \%$ of the cells were stained.

\section{Results}

Macroscopic features (Table I). Macroscopically, two lesions were located in the pancreas body or tail, and three were located in the head. The longitudinal tumor size of the main lesion in the main duct ranged from 2.2 to $4.5 \mathrm{~cm}$ (mean, 3.1 ). The main duct was cut longitudinally. The cut surface showed two distinct patterns. Type I tumors (cases 1, 3, and 5) showed a soft papillary epithelium (Fig. 1A) with intraductal nodular growth (Fig. 1A, arrow). Flat components were observed around the periphery of the tumor (Fig. 1A arrowhead). The surrounding pancreatic parenchyma was normal, with the exception of slight fibrosis. Type II tumors (cases 2 and 4) showed a white, slightly firm tumor in the markedly dilated main pancreatic duct (Fig. 1B). The surrounding pancreatic parenchyma showed firm thickening (arrow).

Microscopic features of tumors in the main duct (Table II). Intraductal epithelial growth in the main duct was observed in all cases and corresponded to the macroscopic features. All tumors showed mucin production. In the main duct, IPMC components were observed in three cases (cases 1, 2, and 4) (Fig. 2A-H). Of these, one case (case 1) also showed adenoma components with gastric-type morphology, and transition from adenoma to intestinal-type carcinoma was observed (Fig. 2A-C). Two cases (cases 3 and 5) did not show IPMC components but showed adenoma or borderline lesions (Fig. 2I and J). The histologic subtype of the tumors in the main duct was determined according to Fukushima et $a l$ and included intestinal-type in one case (case 3), pancreatobiliary-type in one case (case 4) (Fig. 2G and H), and oncocytic-type in one case (case 2) (Fig. 2D-F). In one 


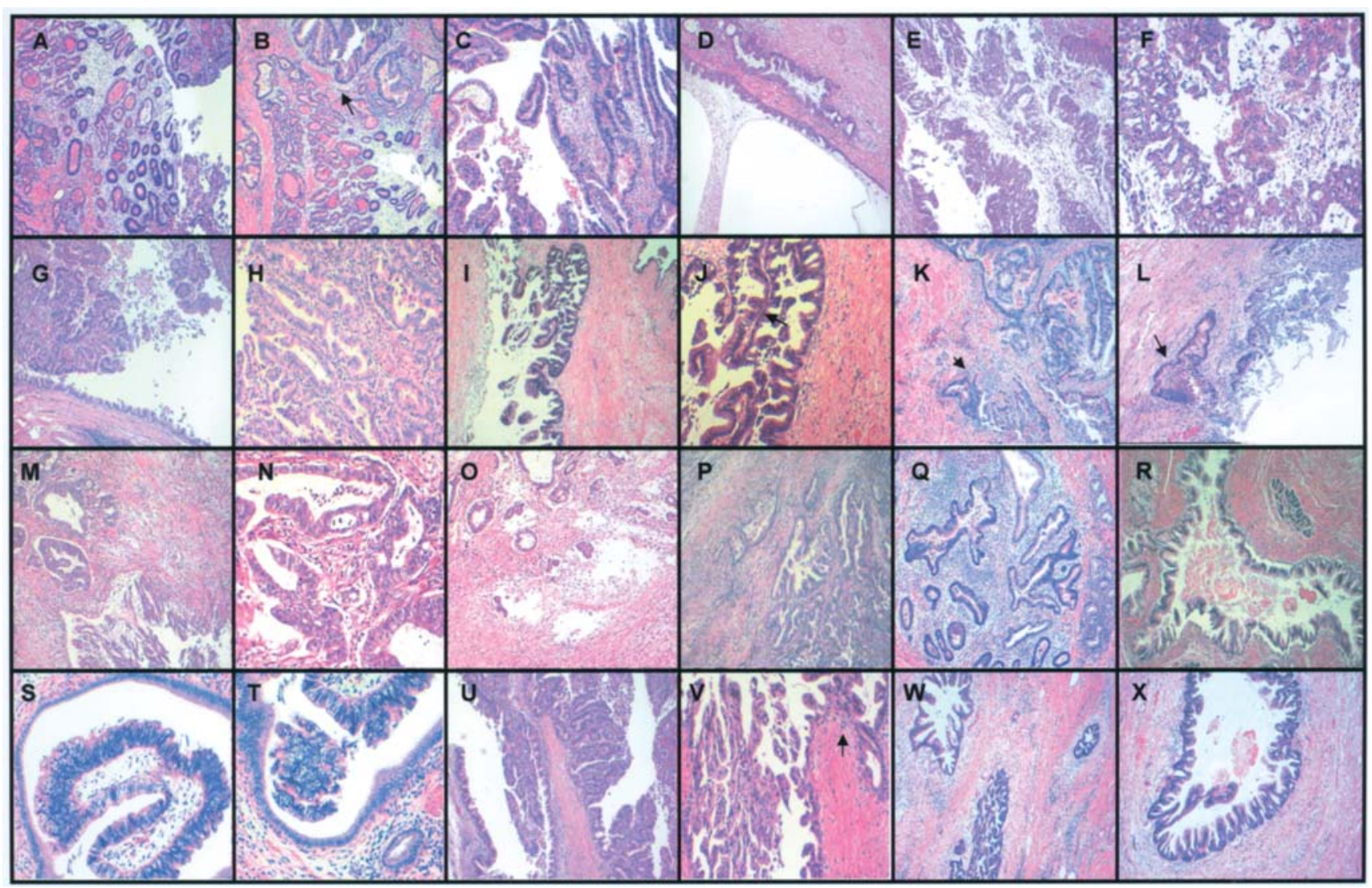

Figure 2. Representative histologic images of resected IPMC. Original magnification is x100 unless otherwise stated. (A-J) The lesions and tumor components in the main duct. (A) Gastric-type adenoma (case 1). Clear columnar cells with basally oriented nuclei show papillary structures. Intraluminal-mucin production is visible. Pyloric gland-like structures are visible in the basal area. Tumor cells show no atypia. (B) Transition between gastric-type adenoma and intestinal-type carcinoma (arrow, case 1). (C) Intestinal-type carcinoma (case 1). Irregular papillary structures consisting of cells with spindle nuclei. Cells show marked atypia and pseudostratification. (D) Main pancreatic duct around tumor components lined with normal epithelial cells (case 2). (E) Normal epithelial cells abruptly replaced by carcinoma in situ (CIS). The epithelium shows marked loss of polarity, cribriform growth, and budding off of cells (case 2). (F) Characteristic features of oncocytic-type CIS (case 2, x200). Epithelium consists of cells with abundant, intensely eosinophilic cytoplasm and shows complex thick papillae with intraepithelial lumina. (G) Normal epithelium abruptly replaced by CIS (case 4). (H) Tumor consisting of cells resembling cholangiopapillary neoplasia shows complex, thin branching papillae with severe atypia corresponding to pancreatobiliary-type CIS. The nodular lesion is composed of an arborizing papillotubular proliferation of cuboidal cells with round nuclei (case 4, x200). (I) Neoplastic epithelium composed of eosinophilic cells with pseudostratified oval- to spindle-shaped nuclei and showing a villous papillary configuration reminiscent of colorectal villous adenoma (case 3 ). (J) An arborizing structure (arrow, case 3, x200). (K-P) Lesions of invasive components. (K and L) Tubular invasive lesions limited to the parenchyma adjacent to the basal membrane (arrow, case 1). (M) Expansive tubular invasion around the CIS components, (arrow, case 2). (N) Tumor cells showing a mixture of medium-sized, duct-like, and tubular structures embedded in a desmoplastic stroma. Incompletely formed glands are visible, corresponding to moderately differentiated adenocarcinoma (case 2, x200). (O) Mixed muconodular-type invasion (case 2). (P) Tubular invasion around the main duct (case 4). (Q) Lowgrade PanIN-complex is visible around the tumor components (case 2). (R) A PanIN-1B lesion with intraluminal mucin (case 3, x200). (S-U) Branch ducts around the main duct, in which neoplastic cells show intraductal nodular growth, abruptly replacing the normal epithelium [(S and T) case 1, $\mathrm{x} 200$; (U): case 4]. These components are considered to represent cancerization of branch ducts. (V) A branch duct filled with neoplastic cells showing complex arborizing structures resembling pancreatobiliary-type IPMN, communicating minimally invasive components (arrow, case 5, x200). (W) High-grade PanIN visible adjacent to the cancerization of a branch duct (case 3). (X) High-grade PanIN with fibrous core (case 3, x200).

case, two subtypes, including gastric- and intestinal-type were mixed (Fig. 2A-C). In case 1, gastric-type adenoma was gradually replaced by intestinal-type carcinoma (Fig. 2B, arrow). In cases 2 and 4, neither adenoma nor borderline lesion was detected, and abrupt transition from normal epithelium to carcinoma was observed in the peripheral area of the tumor (Fig. 2D and G). IPMC in the main pancreatic duct showed invasive components in association with noninvasive components in three cases (cases 1, 2, and 4). In one case (case 1), invasive components were limited to the area of the basal membrane of the main duct, which was considered minimal invasion (Fig. $2 \mathrm{~K}$ and L, arrow). The other two cases (cases 2 and 4) showed massive invasion of the surrounding pancreatic parenchyma (Fig. 2M-P). In the invasive area, tubular carcinoma was the major component in two cases (cases 1 and 4), and tubular carcinoma and mucinous carcinoma were mixed in one case (case 2).

Microscopic features of tumorous components of the branch ducts (Table II). In one case (case 2), branch ducts were not visible, replaced by tumor cells and fibrosis. In the other four cases, a low-grade PanIN-complex was observed around the tumor (Fig. 2Q). Some ducts showed marked dilation and intraluminal mucin (Fig. 2R). These four cases were also accompanied by cancerization of the branch ducts. In two of these cases (cases 1 and 4), normal epithelium was abruptly replaced by neoplastic cells forming papillary to nodular structures (Fig. 2S-U). In the other two cases (cases 3 and 5), 
Table III. Immunohistochemical findings for MUC2.

\begin{tabular}{lccccc}
\hline Case & 1 & 2 & 3 & 4 & 5 \\
\hline Main duct tumor & & & & & \\
$\quad$ Ad-bor & $(-)$ & $*$ & $(++)$ & $*$ & $(++)$ \\
$\quad$ Carcinoma & $(+)$ & $(-)$ & $*$ & $(-)$ & $*$ \\
Invasive components & $(-)$ & $(-)$ & $*$ & $(-)$ & $(-)$ \\
Cancerization of duct & $(-)$ & $(-)$ & $(-)$ & $(-)$ & $(-)$ \\
Low-grade PanIN & $(-)$ & $*$ & $(-)$ & $(-)$ & $(-)$ \\
High-grade PanIN & $*$ & $*$ & $(++)$ & $(-)$ & $(++)$ \\
\hline
\end{tabular}

Ad-bor, adenoma to borderline lesion; Cancerization of duct, cancerization of branch ducts; $(+)$, focally positive; $(++)$, diffusely positive.
Table IV. Immunohistochemical findings for OPRT.

\begin{tabular}{lccccc}
\hline Case & 1 & 2 & 3 & 4 & 5 \\
\hline $\begin{array}{l}\text { Main duct tumor } \\
\quad \text { Ad-bor }\end{array}$ & $(-)$ & $*$ & $(-)$ & $*$ & $(-)$ \\
$\quad$ Carcinoma & $(-)$ & $(++)$ & $*$ & $(++)$ & $*$ \\
Invasive components & $(-)$ & $(++)$ & $*$ & $(-)$ & $(+)$ \\
Cancerization of duct & $(-)$ & $*$ & $(-)$ & $(-)$ & $(-)$ \\
Low-grade PanIN & $(-)$ & $*$ & $(-)$ & $(-)$ & $(-)$ \\
High-grade PanIN & $*$ & $*$ & $(-)$ & $(-)$ & $(+)$ \\
\hline
\end{tabular}

Ad-bor, adenoma to borderline lesion; Cancerization of duct, cancerization of branch ducts; (+), focally positive; (++), diffusely positive.
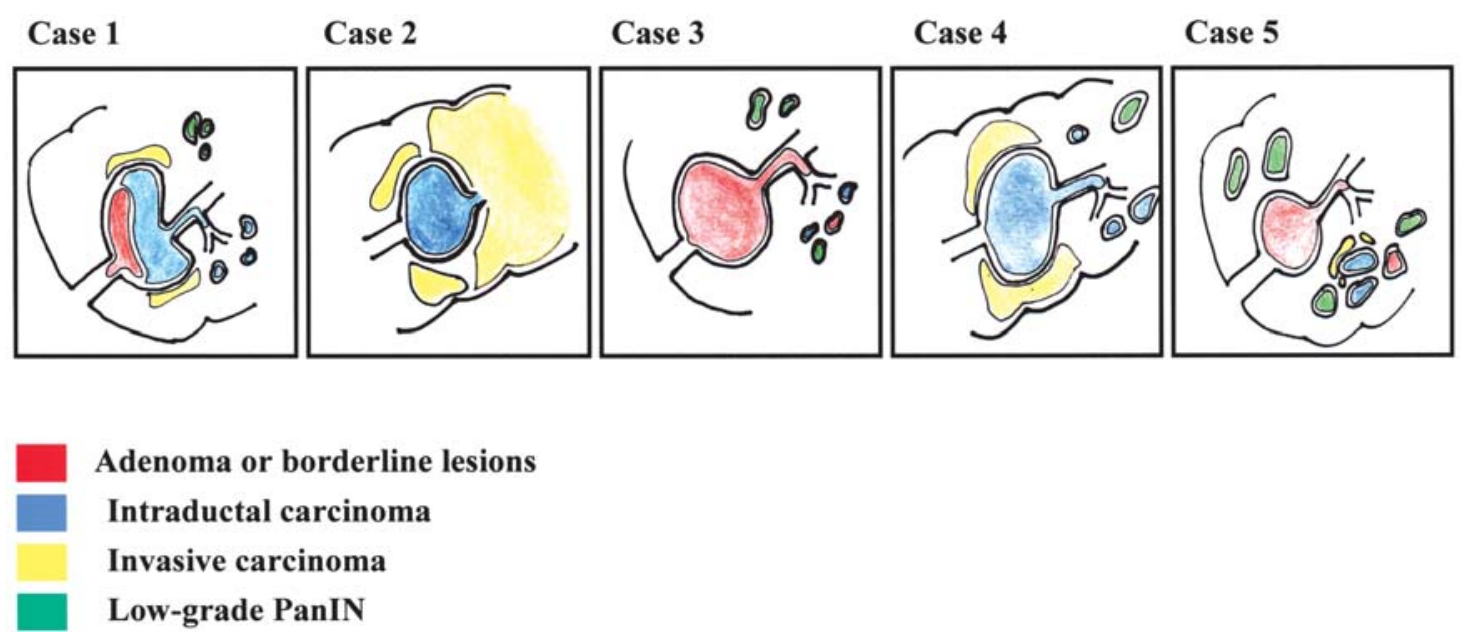

Figure 3. Schematic of histologic distribution in the five cases of IPMC. Each component is mapped in the color indicated.

the branch duct epithelium was filled with arborizing neoplastic papillae, resembling pancreatobiliary-type (Fig. 2V). Cases 3 and 5 also showed neoplastic branch ducts corresponding to high-grade PanIN (Fig. 2W and X). Case 5 showed minimal invasion communicating with the branch duct cancerization (Fig. 2V, arrow). A schematic of the histologic features of each case is shown in Fig. 3.

MUC2 staining (Table III). We evaluated MUC2 immunoreactivity of the intraductal and invasive component $\mathrm{s}$ separately. In the main duct, intestinal-type adenoma and borderline lesions showed diffuse cytoplasmic MUC2 staining (Fig. 4A), whereas IPMC showed no MUC2 staining (Fig. 4B and C) except in one case (case 1), in which focal staining was observed around nuclei in the intestinal-type carcinoma (Fig. 4D and E). In the branch ducts, normal epithelium, low-grade PanIN, and duct cancerization showed no MUC2 staining. However, some areas of high-grade PanIN (in cases 3 and 5) showed diffuse MUC2 staining throughout the epithelial cells (Fig. 4F). When low-grade PanIN and high-grade PanIN were combined in the same duct, the boundary was clearly visible by MUC2 staining (Fig. 4G). None of the invasive components showed MUC2 staining.
OPRT staining (Table IV). In the main duct, intestinal-type adenoma and carcinoma showed no OPRT staining (Fig. 5A and B). Two cases of oncocytic-type (Fig. 5C) and pancreatobiliary-type (Fig. 5D-G) showed OPRT staining in the main duct. In invasive components, one case showed diffuse OPRT staining throughout the neoplastic tubules (Fig. 5H). Neither low-grade nor high-grade PanIN showed OPRT staining (Fig. 5I). Only in case 5 did some areas of PanIN-3 and adjacent minimally invasive components show granular OPRT staining (Fig. 5J).

\section{Discussion}

In the main pancreatic duct, three of the five cases (cases 1, 3 , and 5) showed intestinal-type components, and two of these (cases 3 and 5) did not show CIS. The third (case 1) showed CIS with minimal tubular invasion. The remaining two cases (cases 2 and 4) showed an abrupt transition from normal epithelium to CIS without adenoma components and were accompanied by massive invasion. Although a small number of cases was evaluated in the present study, histologic features included all of the four subtypes described in the recently established consensus criteria for IPMN (1). Therefore, it was reasonable to determine the growth pattern 


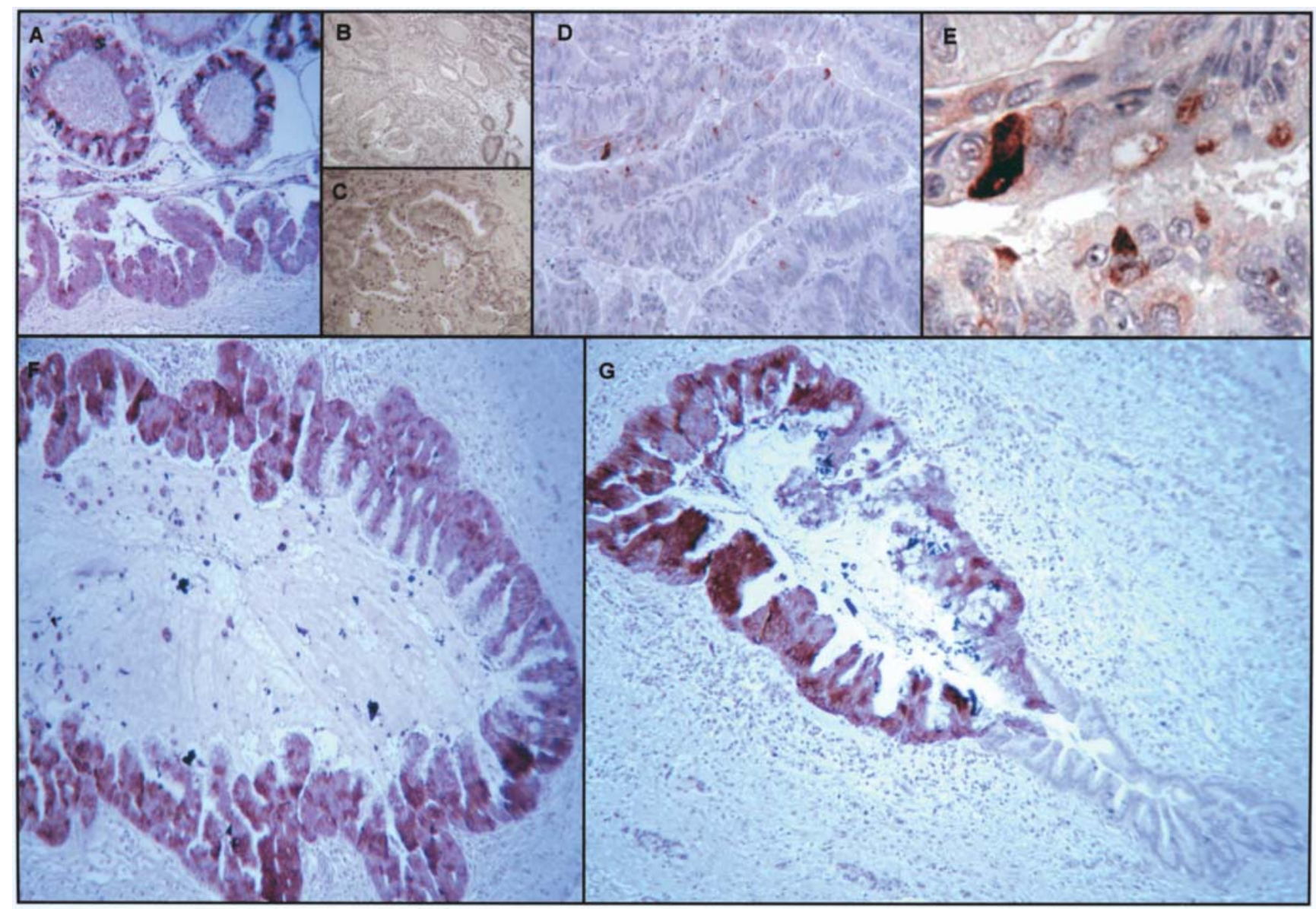

Figure 4. Immunohistochemical staining for MUC2 in IPMCs. Original magnification is x100 unless otherwise stated. (A) In the main pancreatic duct, intestinal-type adenoma shows diffuse MUC2 staining throughout the epithelial cells (case 3). (B) Gastric-type adenoma (case 1) and (C) pancreatobiliarytype IPMC (case 4) do not show MUC2 staining. (D) Focal staining in intestinal-type IPMC (case 1). (E) Focal, granular staining (case 1, x200). (F) Highgrade PanIN around the tumor showing strong MUC2 staining in the branch duct (case 3). (G) In a single duct, high-grade PanIN and low-grade PanIN are mixed, with strong staining localized in the high-grade PanIN (case 5, x200).

of main duct-type IPMC in relation to histologic subtype in these cases. In the four subtypes of IPMN described by Fukushima et al, gastric-type IPMN tends to show low-grade atypia corresponding to intraductal papillary-mucinous adenoma, whereas the other types generally show high-grade atypia corresponding to borderline lesions or CIS (1). In addition, the gastric type usually presents as relatively small cystic lesions in peripheral branch ducts. However, in the present study, one case (case 1) showed gastric-type components in the main duct. In this case, in the peripheral area of the main lesion, gastric-type adenoma showed papillary growth with intraluminal-mucin production and pyloric gland-like structures, whereas intestinal-type CIS was observed with marked intraductal nodular growth in the center area of the main lesion. The transition between each subtype was visible. Ban et al reported that $98 \%$ of gastrictype IPMN are located in branch ducts, and gastric- and intestinal-type IPMNs have distinct biologic pathways (3). Others hypothesize that gastric-type IPMN progresses to intestinal-type IPMN $(1,9)$. Histologic features of our case suggest that gastric-type IPMN can be a precursor of intestinal-type IPMN in the main duct. The other four cases included intestinal-type adenoma in two cases (case 3 and 5), pancreatobiliary (PB)-type CIS in one case (case 4), and oncocytic (Onc)-type CIS in one case (case 2). In the PBand Onc-type, normal epithelial cells were abruptly replaced by CIS, with no adenoma or borderline lesions. PB- and Onctypes are classified as CIS. Hibi et al reported that these types show poor prognosis compared to gastric-and intestinal-types. Massive tubular invasion was also present in case 2 and case 4 (4). Our observations suggest that carcinogenesis without the adenoma-carcinoma sequence in these two types reflects greater invasive status. Several clinical studies have indicated that the presence of invasive components is a predictive factor for poor outcome in IPMN (10-13). Hibi et al also reported that assessment of preoperative pancreatic juice cytology provides findings consistent with the histologic subtype (4). When invasive components cannot be detected radiologically, identification of subtype by cytologic assessment is thus useful for evaluation of IPMN invasiveness.

MUC2 IHC of the main lesions in the five cases of main duct-type IPMC showed diffuse staining in intestinal-type adenoma (case 3 and 5). PB- and Onc-type CIS showed no MUC2 staining, in agreement with previous reports $(2,3)$. However, one of our cases (case 1) showed focal MUC2 staining of intestinal-type CIS components. Nagata et al reported that IPMC shows a low rate of MUC2 expression 


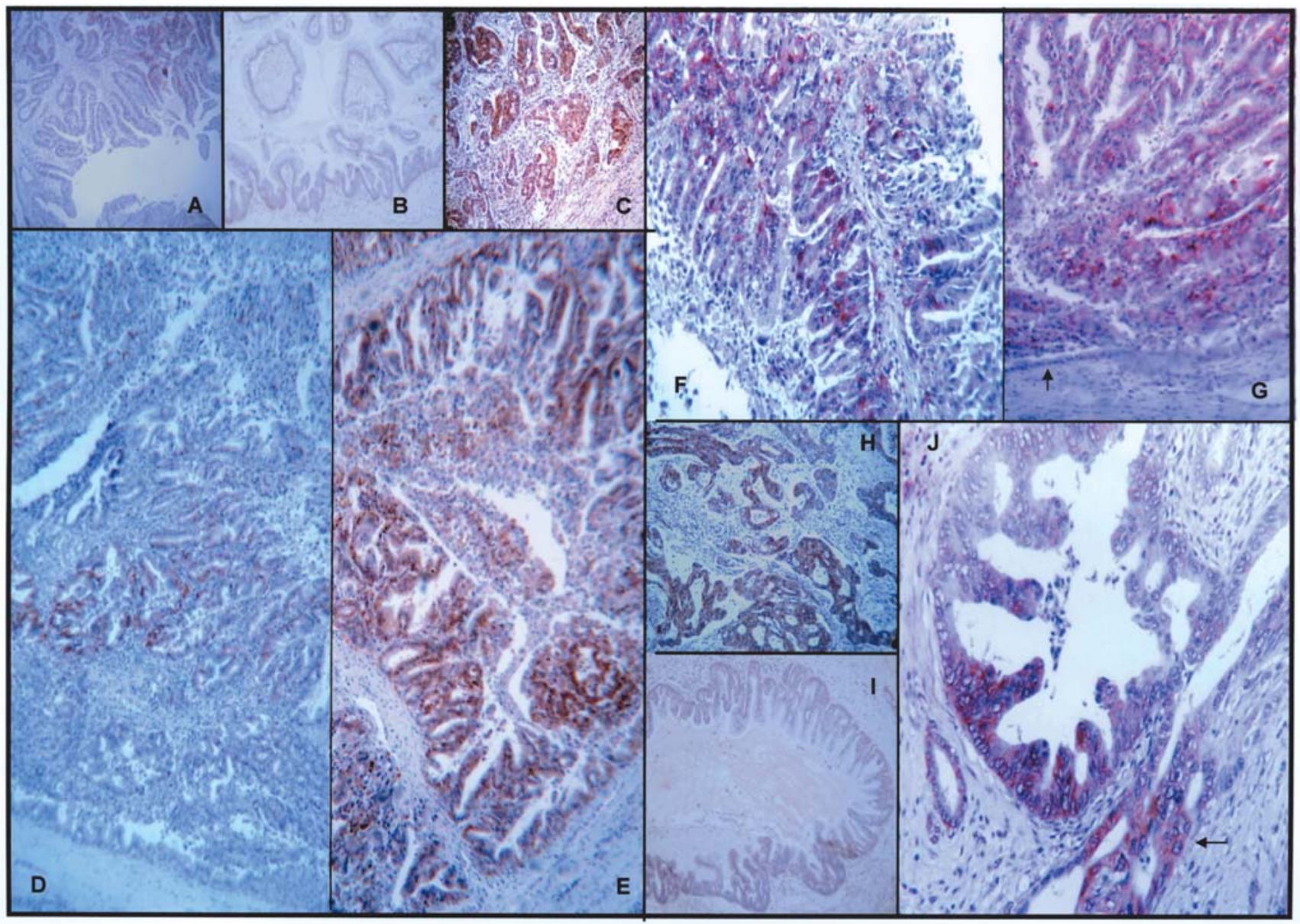

Figure 5. Immunohistochemical staining for OPRT in IPMCs. Original magnification is x100 unless otherwise stated. (A) Intestinal-type carcinoma and gastric-type adenoma do not show OPRT staining (case 1). (B) Intestinal-type adenoma does not show OPRT (case 3). (C) Diffuse staining in oncocytic-type IPMC (case 2). (D-G) In pancreatobiliary-type IPMC, strong cytoplasmic staining is observed, whereas the corresponding normal epithelium does not show OPRT staining (G, arrow). (H) Tubular invasive components show diffuse OPRT staining (case 2). (I) High-grade PanIN with MUC2 staining, shows no OPRT staining (case 3). (J) In case 5, high-grade PanIN and a communicating invasive tubule (arrow) show diffuse OPRT staining.

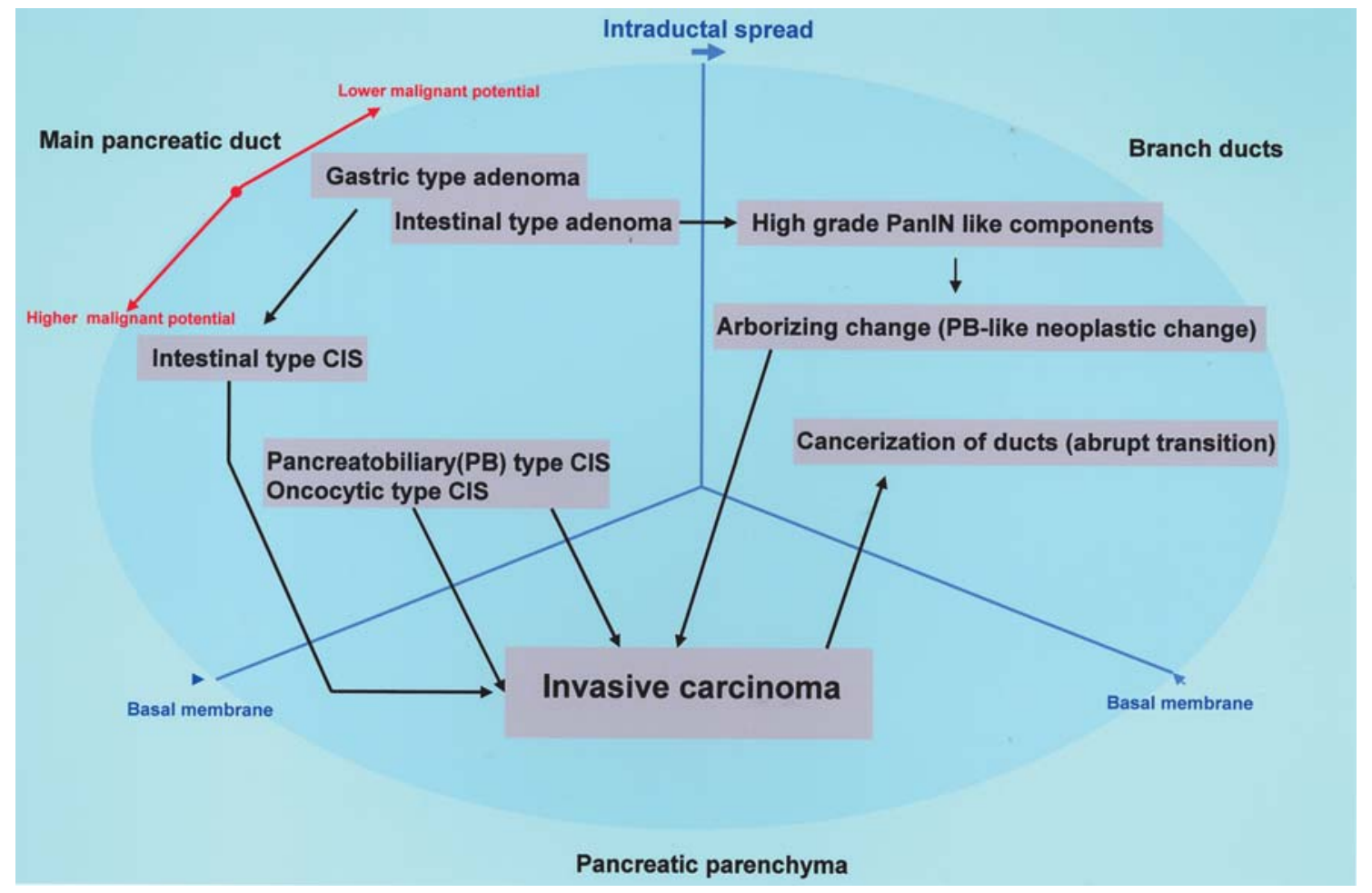

Figure 6. Schematic presentation of the progression of IPMC. 
(3\%) (14). However, Nakamura et al reported positive MUC2 expression in both main and invasive lesions of intestinal-type IPMC (5). Most MUC2-positive components showed diffuse staining in previous reports $(2,3,5)$. In our case, approximately $10 \%$ of intestinal-type CIS components, including cells other than the goblet cells showed MUC2 staining (Fig. 4D and E). This focal MUC2 staining pattern has not been reported previously. In Nakamura et al, invasive carcinoma components positive for MUC2 were mucinous (colloid type) (5). In our case, intestinal-type CIS showed minimal invasion with tubular-type morphology. Colloidtype invasion has lower malignant potential than does tubular-type invasion (15). The prognosis of IPMC showing tubular-type invasion is similar to that of ordinary invasive pancreatic carcinoma (12). Therefore, it is probable that a gradual reduction of MUC2 expression is associated with the stepwise progression of intestinal-type IPMN from adenoma to CIS, through tubular adenocarcinoma (not mucinous carcinoma). To address this hypothesis, an accumulation of resected cases and careful immunohistochemical investigation is necessary.

Branch duct-type IPMN is diagnosed when imaging studies or macroscopic evaluation show cystic dilation $>1 \mathrm{~cm}$ in size located in the secondary duct (16). We observed neoplastic changes in the branch ducts around the main lesion in each case. However, neoplastic changes of branch ducts were detected only by microscopy. Therefore, our five cases should be diagnosed as main duct-type IPMN, not as combined-type IPMN. The neoplastic changes in the branch ducts in our cases can be defined as intraductal involvement of the main lesion, CIS or invasive lesions. We observed three kinds of neoplastic change morphology in the branch ducts: i) abrupt transition from the normal epithelium to neoplastic cells showing intraductal growth; ii) branch duct epithelium filled with arborizing neoplastic papillae, resembling PB-type; ii) high-grade PanIN-like ducts with fibrovascular cores and positive MUC2 staining.

Abrupt transition from normal epithelium to neoplastic cells in the branch ducts has been reported in ordinary pancreatic ductal carcinoma (17). Hruban et al stated that such abrupt transition in branch ducts can be interpreted as secondary infiltration from invasive carcinoma (18). In the present study, tubular invasive components were also observed adjacent to branch ducts showing abrupt transition. Therefore, it is inferred that abrupt transition in branch ducts is due to infiltration from tubular invasive components, not to intraductal spread from CIS. Our observations suggest that IPMC with tubular invasion shows a growth pattern resembling that of pancreatic ductal carcinoma.

High-grade PanIN-like ducts were observed in two of the present cases (cases 3 and 5), in which corresponding main lesions in the main duct were defined as intestinal-type adenoma. Morphologically, there are two explanations for the appearance of high-grade PanIN-like ducts. In one, branch ducts show stepwise progression from normal ducts to low-grade PanIN through high-grade PanIN, resembling that of ordinary pancreatic ductal carcinoma. In the other, main lesions in the main duct show intraductal growth without invasion, consequently forming high-grade PanINlike epithelium in the branch ducts. In our two cases (cases 3 and 5), high-grade PanIN-like ducts showed fibrovascular core (Fig. 2X) and diffuse MUC2 staining, suggesting that high-grade PanIN-like ducts result from the intraductal spread of the main lesion along the ductal epithelium. MUC2-positive PanIN is reported to be a precursor of mucinous carcinoma $(9,19)$. However, the histogenesis differs from that in our observations. In our cases (cases 3 and 5), the main lesions in the main duct were intestinal-type adenoma. Therefore, malignant potential increased during intraductal spread from the main duct to the branch ducts. When low-grade and high-grade PanIN-like epithelia were observed in a single duct, the boundary was clearly visible by MUC2 IHC (Fig. 4G), suggesting that MUC2 IHC can be used to differentiate intraductal spread of intestinal-type IPMN from ordinary PanIN. In addition, our cases (cases 3 and 5) showed characteristic morphologic changes of the branch ducts. Some branch ducts showed arborizing papillotubular proliferation with cells showing severe atypia, resembling PB-type IPMN. These types of ducts were observed adjacent to high-grade PanIN-like ducts. Therefore, we believe that high-grade PanIN-like lesions progress during intraductal spread to ducts showing arborizing proliferation. This morphologic feature has not been described. In our cases (cases 3 and 5), invasive components were not present throughout the tumor, with the exception of minimal invasion communicating a high-grade PanIN-like duct in one case (case 5). These observations indicate that main duct-type IPMC showing intraductal growth to secondary ducts, has low malignant potential. In such cases, the main lesion in the main duct includes an adenoma component of gastric or intestinal subtype. The main ducttype IPMC with no intraductal growth has high malignant potential. In such cases, the main lesion in the main duct shows abrupt transition from normal epithelium to CIS of pancreatobiliary or oncocytic subtype (Fig. 6).

We recently reported that the frequency and strength of OPRT staining significantly increases during the progression of PanIN grade in pancreatic ductal adenocarcinoma $(7,8)$. However, OPRT IHC in the five present cases of main ducttype IPMC, showed that most high-grade PanIN-like ducts were negative for OPRT staining, but showed diffuse MUC2 staining, suggesting that IPMC has a different molecular mechanism from ordinary pancreatic ductal adenocarcinoma. In addition, PB- and Onc-type IPMC showed diffuse OPRT staining, which was maintained in corresponding invasive components in the Onc-type. OPRT expression may be specific for certain IPMC subtypes. Additional studies with larger numbers of cases are necessary to confirm this.

\section{References}

1. Furukawa T, Kloppel G, Adsay NV, Albores-Saavedra J, Fukushima N, et al: Classification of types of intraductal papillary-mucinous neoplasm of the pancreas. Virchows Arch 447: 794-799, 2005.

2. Adsay NV, Merati K, Basturk O, Iacobuzio-Donahue C, Levi E, et al: Pathologically distinct types of epithelium in intraductal papillary mucinous neoplasms. Delineation of an intestinal pathway of carcinogenesis in the pancreas. Am J Surg Pathol 28: 839-848, 2004.

3. Ban S, Naitoh Y, Mino-Kenudson M, Sakurai T, Kuroda M, et al: Intraductal papillary mucinous neoplasm (IPMN) of the pancreas: its histopathologic difference between 2 major types. Am J Surg Pathol 30: 1561-1569, 2006. 
4. Hibi Y, Fukushima N, Tsuchida A, Sofuni A, Itoi T, et al: Pancreatic juice cytology and subclassification of intraductal papillary mucinous neoplasms of the pancreas. Pancreas 34: 197-204, 2007.

5. Nakamura A, Horinouchi M, Goto M, Nagata K, Sakoda K, et al: New classification of pancreatic intraductal papillary-mucinous tumour by mucin expression: its relationship with potential for malignancy. J Pathol 197: 201-210, 2002.

6. Ban S, Naitoh Y, Ogawa F, Shimizu Y, Shimizu M, et al: Intraductal papillary mucinous neoplasm (IPMN) of the gastrictype with focal nodular growth of the arborizing papillae: a case of high-grade transformation of the gastric-type IPMN. Virchows Arch 449: 112-116, 2006.

7. Sanada Y, Yoshida K, Ohara M and Tsutani Y: Expression of Orotate phosphoribosyltransferase (OPRT) in hepatobiliary and pancreatic carcinoma. Pathol Oncol Res 13: 105-113, 2007.

8. Sanada Y and Yoshida K: Immunohistochemical analyses in intraductal gradually transitional components is useful for evaluation of stepwise progression of pancreatic intraepithelial neoplasia. Pancreas 35: 101-103, 2007.

9. Takaori K: Current understanding of precursors to pancreatic cancer. J Hepatobiliary Pancreat Surg 14: 217-223, 2007.

10. Cuillerier E, Cellier C, Palazzo L, Deviere J, Wind P, et al: Outcome after surgical resection of intraductal papillary and mucinous tumors of the pancreas. Am J Gastroenterol 95: 441-445, 2000

11. Marie F, Hammel P, Terris B, Paye F, Scoazec J-Y, et al: Prognosis of malignant intraductal papillary mucinous tumours of the pancreas after surgical resection. Comparison with pancreatic ductal adenocarcinoma. Gut 51: 717-722, 2002.
12. Angerica MD, Brennan MF, Suriawinata AA, Klimstra D and Conlon KC: Intraductal papillary mucinous neoplasms of the pancreas. An analysis of clinicopathologic features and outcome. Ann Surg 239: 400-408, 2004.

13. Sohn TA, Yeo CJ, Cameron JL, Hruban RH, Fukushima N, et al: Intraductal papillary mucinous neoplasms of the pancreas. An update experience. Ann Surg 239: 788-799, 2004.

14. Nagata K, Horinouchi M, Saitou M, Higashi M, Nomoto M, et al: Mucin expression profile in pancreatic cancer and the precursor lesions. J Hepatobiliary Pancreat Surg 14: 243-254, 2007.

15. Adsay NV, Conlon KC, Zee SY, Brennan MF and Klimstra DS: Intraductal papillary-mucinous neoplasms of the pancreas. An analysis of in situ and invasive carcinomas in 28 patients. Cancer 94: 62-77, 2002.

16. Pelaez-Luna M, Chari ST, Smyrk TC, Takahashi N, Clain JE, et al: Do consensus indications for resection in branch duct intraductal papillary mucinous neoplasm predict malignancy? A study of 147 patients. Am J Gastroenterol 102: 1759-1764, 2007.

17. Tanase H, Suda K, Yamasaki S and Nobukawa B: Intraductal low papillary histological pattern of carcinoma component shows intraductal spread in invasive carcinoma of the pancreas. J Hepatobiliary Pancreat Surg 13: 235-238, 2006.

18. Hruban RH, Adsay NV, Albores-Saavedra J, Compton C, Garret ES, et al: Pancreatic intraepithelial neoplasia; a new classification system for pancreatic duct lesions. Am J Surg Pathol 25: 579-586, 2001

19. Albores-Saavedra J, Wu J, Crook T, Amirkhan RH, Jones L, et al: Intestinal and oncocytic variants of pancreatic intraepithelial neoplasia. A morphological and immunohistochemical study. Ann Diagn Pathol 9: 69-76, 2005. 